\title{
A SIMPLIFIED MIXED-CULTURE BIOFILM MODEL
}

\author{
WOLFGANG RAUCH ${ }^{*} \bowtie$, HENK VANHOOREN ${ }^{2}$ and \\ PETER A. VANROLLEGHEM ${ }^{2}$ (4)
}

${ }^{1}$ Department of Environmental Science and Engineering, Technical University of Denmark, Building 115, DK-2800 Lyngby, Denmark and '²IOMATH Department, University Gent, Coupure links 653, 9000 Gent, Belgium

(First received December 1997; accepted in revised form September 1998)

\begin{abstract}
A simple dynamic model is presented for fast simulation of the removal of multiple substrates by different bacterial species growing in a biofilm reactor. The model is an extension to the well-known half-order reaction concept that combines a zero-order kinetic dependency on substrate concentration with diffusion limitation. The basic idea behind this model implementation is to decouple the calculations of the two major processes in the biofilm: substrate diffusion and biochemical conversion. The separate assessment of substrate diffusion allows to relate the penetration depth of substrates to a fraction of biomass that is active in conversion. The conversion is then calculated considering only the active fraction of the biomass. The model is compared to experimental data from the literature and is found to be able to closely replicate the overall dynamics in a biofilm system. The major advantage of the proposed model is the simple structure which leads to a reduction of the computational effort as compared to state-of-the-art mixed-culture biofilm models. (C) 1999 Elsevier Science Ltd. All rights reserved
\end{abstract}

Key words - biofilm, diffusion, modelling, mixed-culture, simulation

\section{NOMENCLATURE}

$A_{F}=$ surface of biofilm $\left(\mathrm{L}^{2}\right)$

$D=$ diffusion coefficient $\left(\mathrm{M}^{2} \mathrm{~T}^{-1}\right)$

$J=$ total transport of substrate through the surface of the biofilm $\left(\mathrm{ML}^{-2} \mathrm{~T}^{-1}\right)$

$L=$ biofilm thickness (L)

$M=$ mass of particulate component (M)

$Q=$ flow in the water phase $\left(\mathrm{L}^{3} \mathrm{~T}^{-1}\right)$

$r=$ reaction rate $\left(\mathrm{ML}^{-3} \mathrm{~T}^{-1}\right)$

$r_{l}=$ reaction rate in the lower part of the biofilm $\left(\mathrm{ML}^{-3} \mathrm{~T}^{-1}\right)$

$r_{u}=$ reaction rate in the upper part of the biofilm $\left(\mathrm{ML}^{-3} \mathrm{~T}^{-1}\right)$

$S=$ concentration of soluble substrate $\left(\mathrm{ML}^{-3}\right)$

$t \quad=$ time $(\mathrm{T})$

$u_{f}=$ velocity by which the surface of the biofilm moves relative to the substratum $\left(\mathrm{MT}^{-1}\right)$

$V=$ volume $\left(\mathrm{L}^{3}\right)$

$X_{F}=$ concentration of bacterial species in the biofilm as biomass per total biofilm volume $\left(\mathrm{ML}^{-3}\right)$

$X_{B}=$ concentration of bacterial species in the biofilm as biomass per bulk liquid volume $\left(\mathrm{ML}^{-3}\right)$

$Y=$ yield coefficient $\left(\mathrm{MM}^{-1}\right)$

$f p=$ fraction of inert material in biomass $\left(\mathrm{MM}^{-1}\right)$

$i_{X}=$ ammonia fraction in biomass $\left(\mathrm{MM}^{-1}\right)$

$\mu=$ maximum growth rate of biomass $\left(\mathrm{T}^{-1}\right)$

*Author to whom all correspondence should be addressed. [Fax: + 454525 2800; e-mail:wr@imt.dtu.dk]. $b=$ decay rate $\left(\mathrm{T}^{-1}\right)$

$k h=$ hydrolysis rate $\left(\mathrm{T}^{-1}\right)$

$k_{\mathrm{d}}=$ detachment rate $\left(\mathrm{MT}^{-1}\right)$

$k_{\mathrm{dt}}=$ detachment coefficient $(-)$

$\beta=$ dimensionless penetration depth of a substrate $(-)$

$\varepsilon=$ fraction of volume occupied by the phase/ component (-)

$\phi=$ active fraction of a species in the biofilm (-)

$\rho=$ density $\left(\mathrm{ML}^{-3}\right)$

$v=$ stoichiometric coefficient $(-)$

\section{Subscripts}

at $=$ suffix denotes attachment

$\mathrm{dt}=$ suffix denotes detachment

$i=$ suffix denotes substrates

$j \quad=$ suffix denotes species

$l=$ liquid phase

$s \quad=$ solid phase

$A=$ autotrophic bacteria

$B=$ bulk liquid

$F=$ biofilm

$H=$ heterotrophic bacteria

$I=$ inert substance

$S=$ biodegradable substance

\section{INTRODUCTION}

The growth of microbial species inside attached biological films causes a significant flux of substrates from the bulk liquid, i.e. the water phase outside of 
the biofilm. As these processes are of great interest for the aquatic ecology in surface waters, for treatment of wastewater and in biotechnology, considerable effort has been made in the last 25 years to develop adequate mathematical models for the description of substrate utilisation and population dynamics in biofilms. The fact that makes such biofilm models relatively complex is that not only the microbial conversion of substrate needs to be considered but also the diffusive transport of soluble substrates inside the biofilm. Early modelling approaches consequently neglected the aspect of biomass growth by assuming a predefined microbial distribution and biofilm thickness (see among others, Williamson and McCarty, 1976; Harremoës, 1978; Mueller et al., 1980). It was not before the mid-80 s that Kissel et al. (1984) and Wanner and Gujer (1986) introduced a more accurate description of the system behaviour, both in time and in space, which made it possible to predict microbial species development over the depth of the biofilm as a function of substrate flux. Recent advances in biofilm research (see among others, Lewandowski et al., 1994; Zhang and Bishop, 1994; Bishop, 1997) conclude that the heterogeneity of the biofilm must also be taken into account. Describing this quantitatively has been attempted in the revised mixedculture biofilm model of Wanner and Reichert (1996).

However, such a detailed mathematical description of the processes in mixed-culture biofilms has also some shortcomings. Most important among these are the required computational effort for solving the resulting set of partial differential equations and the high complexity of the model, which makes accurate estimation of parameter values and initial conditions a tedious task. Hence, rather than following the trend to include more and more phenomena in the model a simpler model is presented here in order to allow fast but sufficiently accurate simulation of biofilm dynamics. The basic idea of the proposed model is to decouple the modelling of the diffusion process and spatial distribution of bacterial species from the biokinetic reactions. This is done by means of a two-step procedure where (1) for each conversion process that is influenced by diffusion, the active fraction of the biomass within the biofilm is computed by means of a simple analytical solution to the problem and (2) all conversions within the biofilm are then calculated as if the biofilm were a ideally mixed reactor but with only the active fraction of the species contributing.

In this article we present the concept and the equations of the simplified mixed-culture biofilm model and discuss the application of the model for two long-term experiments from literature (Horn and Hempel, 1997a,b). The avoidance of the numerical solution of partial differential equations by using the two-step procedure results in a fairly simple model structure. This aspect makes the proposed model an attractive alternative to the existing complex models when emphasis is put more on fast predictions of system behaviour than on detailed understanding.

\section{GENERAL MODELLING CONCEPT}

\section{Background}

Figure 1 illustrates the ideal biofilm system that is mathematically described in the proposed model. To study the interaction between the biofilm compartment and the bulk liquid compartment it is necessary to distinguish between dissolved and particulate constituents. Dissolved substances are transferred from the bulk liquid into the biofilm (the influence of the liquid film diffusion is here neglected, see Christiansen et al., 1995) and then transported by means of molecular diffusion. Particulate components cannot be transferred inside the biofilm but will be adsorbed to the biofilm surface, a process usually denoted as attachment. The reverse phenomenon of displacement of particulate components is consequently addressed as detachment.

The biofilm compartment consists of two phases, that is (1) the liquid phase, in which the dissolved substances are transported due to the diffusion phenomena and (2) the solid matrix, which consists of several species of bacteria as well as of particulate substrate and inert material. Concentration of particulates and density in biofilm is therefore to be expressed as follows:

$$
\varepsilon_{l}+\sum_{j=1}^{n_{X}} \varepsilon_{s j}=1 \quad \text { with } \quad \varepsilon_{s j}=\frac{X_{j}}{\rho_{j}}=\frac{M_{F j}}{V_{F}} \frac{1}{\rho_{j}}
$$

where $\varepsilon_{l}$ is the volume fraction of the liquid phase, $\varepsilon_{s j}$ the volume fraction of the particulate component $j$ in the solid matrix, $n_{X}$ the number of particulate components considered and $\rho_{j}$ the density of the component. The concentration of a specific component $\left(X_{j}\right)$ is given by dividing its mass $\left(M_{F j}\right)$ by the entire volume of the biofilm $\left(V_{F}\right)$, i.e. volume of liquid and solid phase. The density of a specific

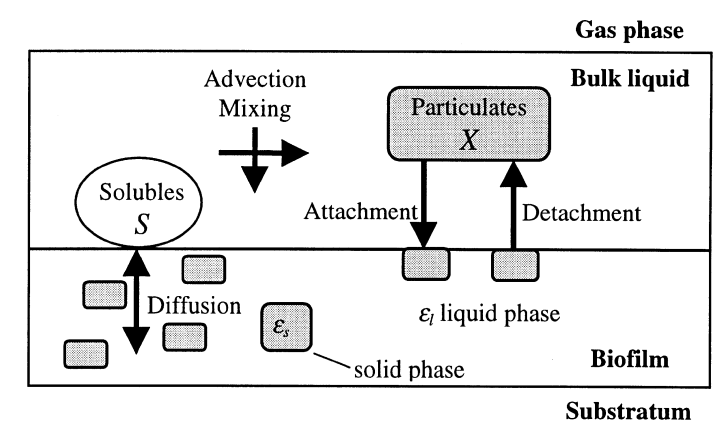

Fig. 1. Transport processes in an ideal biofilm system. 
component $j, \rho_{j}$, must not be confused with the mean biofilm density $\rho_{m}$

$$
\rho_{m}=\sum_{j=1}^{n_{X}}\left(\varepsilon_{s j} \rho_{j}\right)=\frac{\sum_{j=1}^{n_{X}} M_{F j}}{V_{F}}
$$

which can be measured experimentally. For reason of simplification the proposed model is applying the widespread assumption to see the biomass as a continuum (e.g. Wanner and Gujer, 1986), that is $\rho_{j}=$ constant $=\rho_{m} /\left(1-\varepsilon_{l}\right)$.

Mass transport and zero-order reaction in a biofilm-concept of active fractions

Soluble substrate from the bulk liquid is transferred inside the biofilm and then transported further by means of molecular diffusion. The substrate is simultaneously utilised in the film by the bacteria for growth. In case the substrate does not fully penetrate the biofilm, the reaction is considered as diffusion limited, i.e. the reaction is taking place only over a certain depth of the biofilm.

Harremoës (1978) developed analytical solutions for calculating diffusion limited biofilm reactions by adopting mass balance equations for biofilms with an idealised geometry (Fig. 2) and steady state conditions:

$$
\frac{\partial^{2} S}{\partial z^{2}}=\frac{r}{D}
$$

where $S$ is the concentration of the substrate at the location $z$ in the biofilm, $D$ the diffusion coefficient for the substrate and $r$ the volumetric reaction rate (positive for substrate removal). To derive an analytical solution to this second-order differential equation, the reaction rate needs to be defined. Harremoës (1978) pointed out that the specific growth rates of bacteria can be assumed zero-order with respect to the concentration of the substrate $S$ in the biofilm. The reason is that the saturation coefficients (assuming Monod-type kinetics) are very small for most substrates at hand (dissolved oxygen, soluble organic matter, ammonia and nitrate). Hence, the biofilm volume, where the assumption of zero-order kinetics does not hold, is very small and can be conveniently neglected. The volumetric (zero-order) reaction rate of a species with respect to the substrate $S$ can thus be written as:

$$
r=-\mu X v
$$

where $r$ is the zero-order reaction rate for the bacterial species $X$ with respect to the substrate $S, \mu$ the (max) specific growth rate of the species $X$ and $v$ the stoichiometric coefficient.

The penetration depth can be derived from an analytical solution to equation 3 (for details, see Harremoës, 1978):

$$
z=\sqrt{\frac{2 D S_{0}}{r}}
$$

where $S_{0}$ is the concentration of substrate $S$ in the bulk liquid.

The basic consideration is now whether the biofilm is fully penetrated by the substrate or not. In case the biofilm is fully penetrated (no substrate limitation or $z \geqslant L$ ), the solution to the problem is obvious, as the reaction takes place over the full depth of the biofilm $L$ with a constant (zero-order) maximum rate. However, in case of substrate limitation the reaction is taking place only over a certain depth of the biofilm.

Hence, when there is substrate limitation, the biofilm is partitioned in an active (upper) part and an inactive part close to the substratum. This limitation effect can also be expressed by assuming only a certain fraction of the biomass to be active. So, a solution can be given for the flux of substrate $S$ into the biofilm:

$$
J=-\mu X \nu \phi L \text { and } \phi[0,1]
$$

where $J$ is the total transport of the substrate $S$ through the surface of the biofilm, $L$ the biofilm thickness and $\phi$ the active fraction of species $X$.

The underlying idea is that the active fraction of the biomass $\phi$ is equal to the dimensionless penetration depth of the limiting substrate $\beta(z / L)$ :

$$
\beta=\sqrt{\frac{2 D S_{0}}{r L^{2}}}
$$

\section{Assumptions underlying the concept}

The concept of active fractions forms the basis of the simplified mixed-culture biofilm model. It is

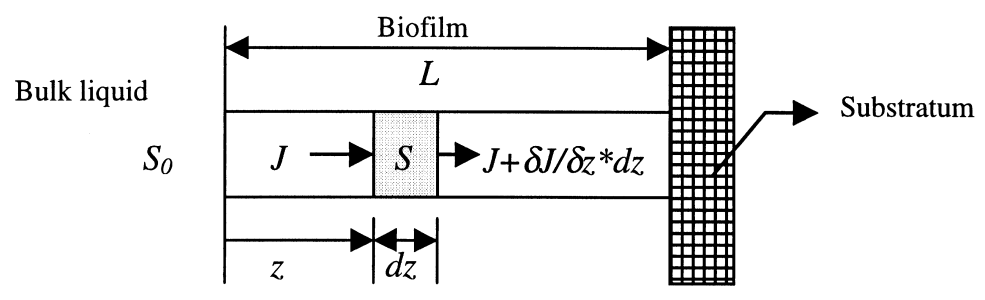

Fig. 2. Idealised biofilm system with uniform spatial distribution of microbial species. 
therefore essential to be aware of the assumptions this model relies upon: (1) the biofilm is assumed to be of homogeneous structure and density, (2) the microbial species in the biofilm are uniformly distributed, (3) the typically modelled stagnant liquid layer is neglected, (4) reaction rates are of zeroorder, (5) soluble components that emerge from conversion processes inside the film are assumed to be subject of immediate out-diffusion at the surface of the biofilm and (6) an instantaneous steady-state substrate profile is assumed.

Assumptions (1) and (2) can be particularly justified as long as the heterotrophic loading to the system under study is not too high (as in the applications described below).

The incorporation of a stagnant layer (see assumption (3)) does not affect the basic assumptions of the model and might therefore be done on occasion. But the possible improvement of the model by including an external diffusion resistance is, however, highly dependent on biofilm loading and fluid velocity. The effect of such effort is most likely rather seen on the decrease of the calculation speed than on the improvement of the predictive power of the model (see Christiansen et al., 1995). As the emphasis of the modelling exercise was on simplification and calculation speed, it was chosen not to incorporate external diffusion resistance.

The use of zero-order reaction rates (assumption (4)) is very widespread in biofilm modelling, as it is a good description of biofilm kinetics when substrate concentrations are sufficiently high. Other reaction rates can also be used as long as they give rise to analytical solutions for the substrate penetration depth as this is inherent to the decoupling of diffusion and biokinetic reaction in this approach.

Assumption (5) is only of importance when a soluble component is produced inside the biofilm due to conversion processes. The assumption of immediate out-diffusion neglects the possible accumulation of the component inside the biofilm. However, due to the principle of homogeneous species distribution in the biofilm (assumption (2)) this effect is compensated in the model: the out-diffusion leads to an immediate increase of the concentration of the component in the bulk liquid and, subsequently, to an increase of the substrate flux into the biofilm.

Assumption (6) is, in principle, violating the mass balances in a dynamic situation and therefore needs to be discussed further. As outlined in Kissel et al. (1984) the characteristic time of the film diffusion phenomena is about one order of magnitude smaller than the time scale of reactions in the film. From the point of view of the dynamics in the conversion processes it can therefore be assumed that the substrate profile reaches an equilibrium very fast. This offers the possibility to omit consideration of the dynamics of the diffusion process and model diffusion as a steady state phenomenon within each time step (by means of an analytical solution). The error that is introduced by this approach to the mass balances in the model is assumed negligible.

\section{DIFFUSION LIMITATION IN A MULTIPLE SUBSTRATE/ SPECIES SYSTEM}

Applying the theory of diffusion limitation and zero-order substrate utilisation to biological processes (Harremoës, 1978), it needs to be considered that bacteria generally require multiple substances $\left(S_{i}\right)$ for growth, usually an electron acceptor, an electron donor and nutrients.

Figure 3 outlines the general idea of the theory to the simple example of a 2 substrate/ 1 species system: For growth of heterotrophic bacteria in the biofilm two substrates are required, i.e. an electron acceptor (dissolved oxygen $S_{\mathrm{O}}$ ) and an electron donor (soluble organic matter $S_{\mathrm{S}}$ ). Both substrates are essential for bacterial growth and, consequently, the whole process stops when one of them is not
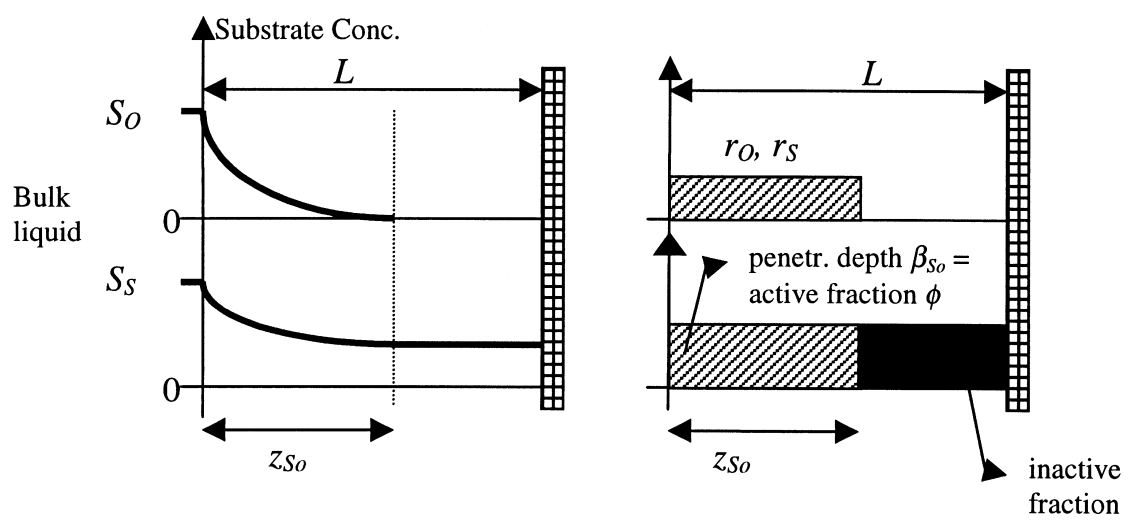

Fig. 3. Illustration of a diffusion limited reaction in a system with two substrates $\left(S_{\mathrm{O}}\right.$ oxygen and $S_{\mathrm{S}}$ organic matter) and one species. $S_{\mathrm{O}}$ is the limiting substrate. 
available. In Fig. 3 oxygen is considered to be the limiting substrate.

In this simple system the dependency of the total reaction rate and of the limitation of bacterial growth on the bulk liquid substrate concentration can be easily derived. Indeed, the active fraction of the biomass $\phi$ is equal to the dimensionless penetration depth of the limiting substrate $\beta_{S_{\mathrm{o}}}\left(z_{S_{\mathrm{o}}} / L\right.$ in Fig. 3). The fluxes of the different substrates $i$ into the biofilm are then derived directly from:

$$
J_{i}=-\mu X v_{i} \phi L \text {. }
$$

However, the straightforward relation $\phi=$ $\beta\left(S_{\text {limiting }}\right)$ only holds for very simple systems as in Fig. 3. A problem arises when this active fraction concept is applied to more complex problems. Indeed, in most cases there are various bacterial species $\left(X_{j}\right)$ present in the biofilm, which sometimes compete for the same substrate (e.g. both heterotrophic and autotrophic bacteria require oxygen as well as ammonia for aerobic growth). On the one hand, equations for the dimensionless penetration depth $\beta_{i}$ can be written that relate to the exhaustion of substrates while, on the other hand, the active fraction $\phi_{j}$ for each biomass species is needed for further calculation. Although there exists a relationship between these two variables, this relation is case specific and requires a thorough analysis of the specific problem at hand, as illustrated below.

In the following, the basic theory is outlined for a system with substrates $S_{i}$ and species $X_{j}$. Based on the considerations above, the volumetric reaction rate of a species with respect to each substrate is written as:

$$
r_{i j}=-\mu_{j} X_{j} v_{i j}
$$

where $r_{i j}$ is the zero-order reaction rate for the bacterial species $X_{j}$ with respect to the substrate $S_{i}, \mu_{j}$ the (max) specific growth rate of the species $X_{j}, v_{i j}$ the stoichiometric coefficient and $i, j$ the suffices denoting the substrates and species respectively. In case different species use the substrate $S_{i}$, the total volumetric reaction rate of this substrate becomes:

$$
r_{i}=\sum_{j}-\mu_{j} X_{j} v_{i j}
$$

Now again, in case the biofilm is fully penetrated, all reactions take place over the full depth of the biofilm $L$ with a constant maximum rate. On the contrary, in case any substrate limitation occurs, the reaction takes place only over a fraction of the total depth of the biofilm.

Given that each reaction is governed by the activity of only one bacterial species, the limitation effect can again be expressed by assuming that only a certain fraction of this particular species to be active. Hence a general solution can be given for the flux of each substrate into the biofilm:

$$
J_{i}=\sum_{j}-\mu_{j} X_{j} v_{i j} \phi_{j} L \quad \text { and } \quad \phi_{j}[0,1]
$$

where $J_{i}$ is the total transport of the substrate $S_{i}$ through the surface of the biofilm, $L$ the biofilm thickness and $\phi_{j}$ the active fraction of species $X_{j}$. The calculation of the active fractions is outlined in the following.

\section{STEP 1: ACTIVE FRACTIONS IN A MULTIPLE SUBSTRATE/SPECIES SYSTEM}

The proposed model is based on a two-step procedure for calculating the conversions in the biofilm reactor in each time-step. First, the diffusion depth of the substrates into the biofilm is found analytically by assuming pseudo-steady-state conditions, zero-order conversion rates and a homogeneous distribution of the bacterial species within the biofilm. The result of this first step is an estimate of the amount of bacterial mass that is actually active in the conversion processes (active fraction). The other, inactive fraction of the bacterial mass is not exposed to the substrate necessary for performing the conversion processes (diffusion limited). Given this information, the conversion of the substrates in the reactor is then calculated in the second step.

Analytical derivation of the penetration depth of substrates

As stated above, Fig. 3 shows the simplest case with respect to diffusion limitation: From the point of view of spatial distribution all processes are stopped when one of the limiting substrates is exhausted. The penetration depth for any substrate, given that all other substrates are in excess, can be derived from an analytical solution to equation 3 :

$$
z_{i}=\sqrt{\frac{2 D_{i} S_{i 0}}{r_{i}}} \text { and } \beta_{i}=\sqrt{\frac{2 D_{i} S_{i 0}}{r_{i} \cdot L^{2}}}
$$

with

$$
r_{i}=\sum_{j}-\mu_{j} X_{j} v_{i j}
$$

where $r_{i}$ is the zero order conversion rate of the substrate $S_{i}$ (taking into account all bacterial species $X_{j}$ that use this substrate under the given environmental condition, i.e. aerobic-anoxic), $\beta_{i}$ is the dimensionless penetration depth of substrate $S_{i}, S_{i 0}$ the concentration of substrate $S_{i}$ in the bulk liquid and $L$ the biofilm thickness.

However, for a multiple substrate/multiple species system the aspect of diffusion limitation can easily become more complex. Generally, sequential diffusion limitation occurs if a substrate $S_{a}$ is used in two (or more) processes and one of these processes is limited earlier than the other by a different substrate $S_{b}$. As a result, the zero-order conversion rate of the substrate $S_{a}$ is no longer the same in the whole biofilm. The penetration depth $z_{a}$ of the sub- 

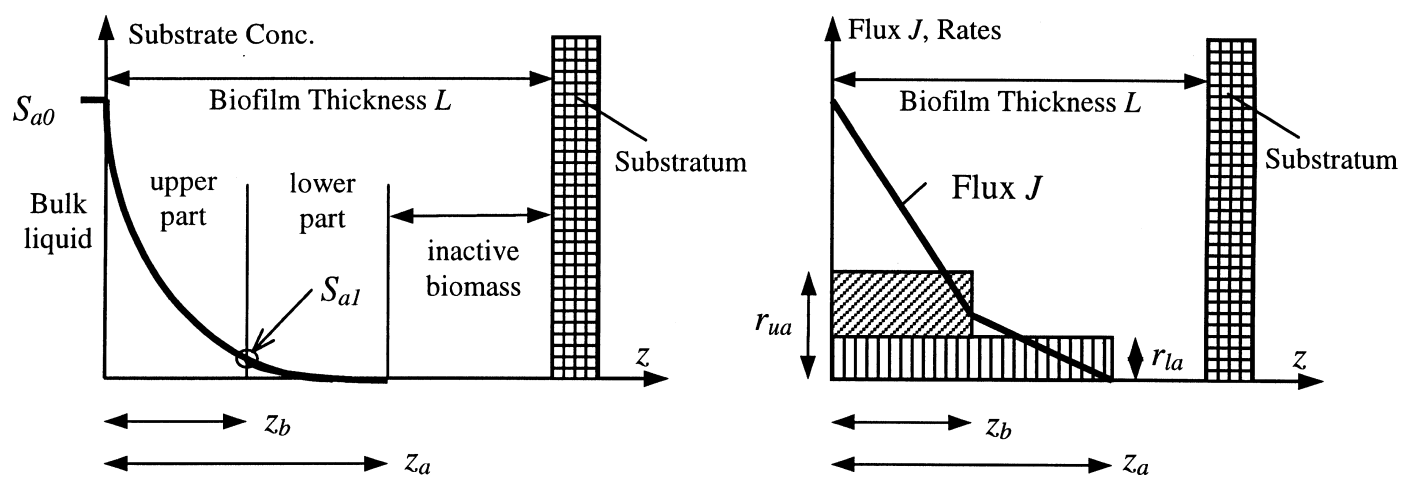

Fig. 4. General description of sequential diffusion limitation.

strate is divided in two parts where the conversion rate in the upper active part $\left(r_{\text {ua }}\right)$ is different (higher) from the one in the lower active part closer to the substratum $\left(r_{\mathrm{la}}\right)$. Indeed, in the upper part two processes consume substrate $a$, while in the lower only one process proceeds. This is outlined generally in Fig. 4.

The depth of the upper part is determined by the penetration depth $z_{b}$ of the substrate $S_{b}$ that is limiting first. Consequently, the flux of the substrate $S_{a}$ deeper into the biofilm is linearly decreasing only within each of the two parts of the biofilm but no longer over the whole penetration depth. Nevertheless, the penetration depths $z_{a}$ and $z_{b}$ can be derived analytically (Rauch and Vanrolleghem, 1997):

$$
\begin{aligned}
& z_{b}=\sqrt{\frac{2 D_{b} S_{b 0}}{r_{b}}} \text { and } \\
& z_{a}=\sqrt{z_{b}^{2}\left(1-\frac{r_{\mathrm{ua}}}{r_{\mathrm{la}}}\right)+\frac{2 D_{a} S_{a 0}}{r_{\mathrm{la}}}}
\end{aligned}
$$

where $r_{b}$ is the zero-order reaction rate in the upper part with respect to the first limiting substrate $S_{b}$ (taking into account all species $j$ that use this substrate under the given environmental condition), $r_{\text {ua }}$ the zero-order reaction rate in the upper part with respect to $S_{a}$ (taking into account all species $X_{j}$ that use this substrate under the given environmental condition), $r_{\mathrm{la}}$ the zero-order reaction rate in the lower part with respect to $S_{a}$ (taking into account all species $X_{j}$ that use this substrate and which are not already limited by another substrate), $S_{b 0}$ the bulk liquid concentration of substrate $S_{b}$ that is lim- ited first and $S_{a 0}$ the bulk liquid concentration of substrate $S_{a}$.

The aerobic growth in a system with both heterotrophic $\left(X_{H}\right)$ and autotrophic bacterial species $\left(X_{A}\right)$ is an example of this sequential diffusion limitation. Growth of the species requires three substrates, i.e. dissolved oxygen $S_{\mathrm{O}}$, soluble organic matter $S_{\mathrm{S}}$ and ammonia $S_{\mathrm{NH}}$. If $S_{\mathrm{S}}$ is limiting, then the growth of heterotrophs stops but the growth of autotrophs proceeds until either oxygen or ammonia gets limiting for them. Hence, this is a sequencing diffusion limitation for oxygen or ammonia. In the outer part of the biofilm (until $S_{\mathrm{S}}$ is utilised) both heterotrophic and autotrophic growth take place simultaneously whereas in the next zone (until any other substrate gets limiting) only the autotrophic growth process utilises $S_{\mathrm{NH}}$ and $S_{\mathrm{O}}$. Figure 4 expresses this situation in general terms.

\section{Calculation of active fractions of bacterial species: an example}

The example used to introduce the approach for the calculation of the active fractions for the penetration depths considers the main carbon/nitrogen cycles in a biofilm, i.e. carbon removal, nitrification and denitrification (given on Peterson matrix representation (Henze et al., 1987) in Table 1). Heterotrophic bacteria are growing under two different environmental conditions in the system, i.e. under both aerobic and anoxic conditions. Other than in continuously stirred tank reactors, a spatial distribution of the oxygen concentration exists in biofilms. Consequently, in biofilms simultaneous nitrification and denitrification can occur, where the top layers of the biofilm are nitrifying

\begin{tabular}{|c|c|c|c|c|c|}
\hline Process & $S_{\mathrm{O}}\left(\mathrm{ML}^{-3}\right)$ & $S_{\mathrm{S}}\left(\mathrm{ML}^{-3}\right)$ & $S_{\mathrm{NO}}\left(\mathrm{ML}^{-3}\right)$ & $S_{\mathrm{NH}}\left(\mathrm{ML}^{-3}\right)$ & Process rate $\left(\mathrm{ML}^{-3} \mathrm{~T}^{-1}\right)$ \\
\hline $\begin{array}{l}\text { Aerobic het. growth } \\
\text { Anoxic het. growth } \\
\text { Aerobic aut. growth }\end{array}$ & $\begin{array}{c}1-1 / Y_{H} \\
1-4.57 / Y_{A}\end{array}$ & $\begin{array}{l}-1 / Y_{H} \\
-1 / Y_{H}\end{array}$ & $\begin{array}{c}-\left(1-Y_{H}\right) / 2.86 Y_{H} \\
1 / Y_{A}\end{array}$ & $\begin{array}{c}-i_{x} \\
-i_{x} \\
-1 / Y_{A}-i_{x}\end{array}$ & $\begin{array}{c}\mu_{H} \cdot X_{H} \\
\mu_{H}^{*} \cdot X_{H} \\
\mu_{A} \cdot X_{A}\end{array}$ \\
\hline
\end{tabular}

Table 1. Matrix representation of aerobic/anoxic growth of heterotrophic/autotrophic bacteria 
whereas the deeper layers denitrify. The spatial distribution is taken into account by means of the anoxic active fraction coefficient $\phi_{H}^{*}$ for heterotrophic bacteria. However, note that heterotrophic bacteria have a preference for oxygen. As a result nitrate can never be the substrate that is limiting heterotrophic activity in the first place, but only after all oxygen is utilised. Furthermore, the biokinetic model outlined in Table 1 assumes that species growth limitation by nitrogen is only due to ammonia exhaustion. The anoxic growth rate is denoted as $\mu_{H}^{*}$.

With respect to utilisation and production of nitrate in the biofilm, two aspects need to be considered. First, a problem arises when more nitrate is produced via nitrification than utilised due to denitrification. As a consequence, a nitrate flux occurs in the opposite direction, i.e. from the biofilm back into the bulk liquid. The transport of the net produced nitrate through the nitrification zone is subject to diffusion. A second problem arises for nitrate utilisation which occurs usually in the deeper layers of the biofilm. Here also, nitrate is transported through a non-reactive layer. The simplified model gives no direct opportunity to consider these diffusion processes and an instantaneous transport of the soluble products is assumed in both cases (assumption (5)). Hence, produced nitrate does not accumulate in the biofilm but is considered only by means of the net flux transport through the biofilm surface.

The procedure to derive limitation of active fractions of the species in the biofilm for each specific situation of substrate can be outlined as follows for this heterotrophic/autotrophic biofilm:

1. For each of the substrates $S_{\mathrm{O}}, S_{\mathrm{S}}, S_{\mathrm{NO}}$ and $S_{\mathrm{NH}}$ the dimensionless penetration depth $\beta_{i}\left(=z_{i} / L\right)$ is calculated from equation 12 . If none of the substrates is limiting the biofilm is fully penetrated and all active fractions $\phi$ are equal to one. If not, the substrate with the smallest value $\beta_{i}$ is limiting first.

2. The actual case of substrate limitation is derived from Fig. 5 where the order of limitation is listed together with the active fractions of the relevant species. Unless $S_{\mathrm{NH}}$ is limiting, another substrate might cause a sequential diffusion limitation that has to be taken into account.

3. If there is no $S_{\mathrm{NH}}$ limitation then the penetration depths of the substrates are calculated from equation 14 based on the possible order of limitations.

4. If any of these dimensionless penetration depths is smaller than one, the corresponding substrate
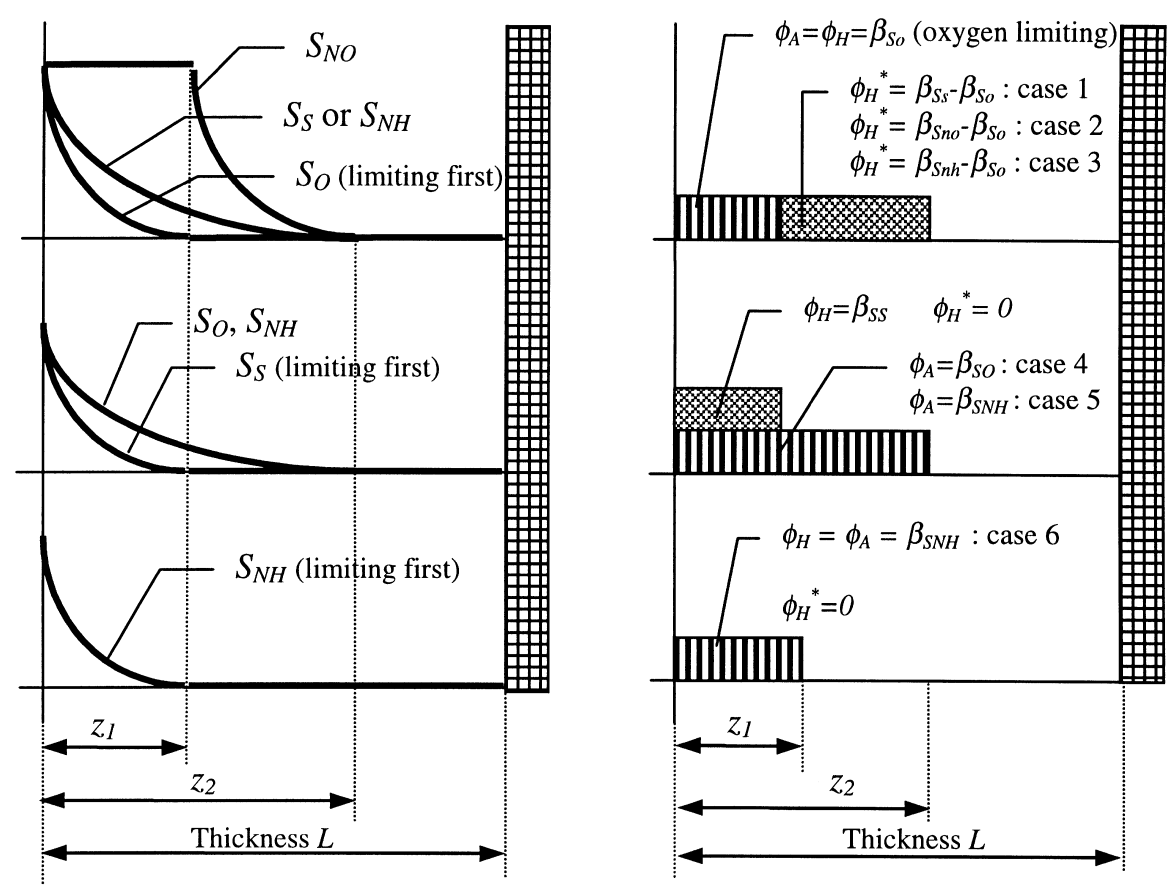

Fig. 5. Six possible situations for substrate limitation in the competition of heterotrophic and autotrophic growth in an idealised biofilm. Left: Substrate profiles and Right: active fraction of bacteria species based on zero-order kinetics. From top to bottom: case 1, 2 and $3=$ oxygen limited in the first place and then either organic matter, nitrate or ammonia limited; cases 4 and 5 = organic matter limited in the first place and then either oxygen or ammonia limited; and case $6=$ ammonia limited. 
is limiting in the second place and the active fraction is obtained from Fig. 5.

\section{General algorithm for calculating active fractions}

The following algorithm is generally applicable for determining the active fractions in a multiple species/substrate systems:

1. Make the process matrix, indicate all negative stoichiometric values and evaluate the sequence of switching from one substrate to another for processes that are "on" or "off" (e.g. anoxic growth is not operational as long as oxygen is "on", assimilation of nitrate as nitrogen source is "off" as long as ammonia is "on", etc.);

2. Calculate the dimensionless penetration depth $\beta$ for each of the relevant components and for all reactions that are "on" assuming zero-order reaction rates and no limitation by any other substrate;

3. The smallest $\beta$ found is retained (unless it is larger than one) and this determines the penetration depth of the limiting substrate;

4. Some reaction(s) may be switched "off" and others "on" by this absence of substrate in the deeper layers of the biofilm;

5. Unless all reactions are "off" or the smallest $\beta$ is larger than one, go to 2 .

\section{STEP 2: OVERALL MODEL FOR THE BULK LIQUID IN CONTACT WITH A BIOFILM}

Concept for describing bulk liquid-biofilm interaction

The procedure outlined above is only the first step for deriving a mathematical description of the biokinetic processes within the biofilm and of the mass exchange between bulk liquid and biofilm compartment. The result of the analysis with respect to diffusion limitation is a quantification of the active mass of each species present in the whole biofilm with respect to the processes. Also the dynamic changes in the bulk liquid need to be considered and the mass transfer between those two phases.

The system is seen in the following as two connected ideally mixed tank reactors where one is representing the bulk liquid and the other the biofilm (Fig. 6). The components in both tanks are expressed differently, in the bulk liquid in terms of concentrations $\left(\mathrm{ML}^{-3}\right)$, as usual, and in the biofilm as mass $M_{F}(\mathrm{M})$. The reason is that the thickness of the biofilm compartment is changing, which can be taken into account more easily by balancing masses than by concentrations. Neglecting the dynamic changes in biofilm density, the thickness of the biofilm can be computed at any time instant from (2)

$$
L=\frac{\sum M_{F j}}{\rho_{m} A_{F}} .
$$

The different dimensions of the particulate components in both reactors (concentrations in the bulk liquid and mass in the biofilm) do not allow a direct interaction of components in both compartments. Traditionally, the formulation of the mass transfer between the phases has to account for that. Alternatively, one can express the reactions in the biofilm as a volumetric reaction rate $r_{B i}$ with respect to the substrate concentration in the bulk liquid. This volumetric reaction rate is derived from equation 11, that is from the areal flux $J_{i}$ of a substrate $S_{i}$ through the surface of the biofilm:

$$
r_{B i}=\frac{J_{i}}{V_{B} / A_{F}}=\frac{A_{F}}{V_{B}} \sum_{j}-\mu_{j} X_{j} v_{i j} \phi_{j} L .
$$

Rewriting this equation gives:

$$
\begin{aligned}
r_{B i} & =\sum_{j}-\mu_{j} v_{i j} \phi_{j} L \frac{M_{F j}}{A_{F} L} \frac{A_{F}}{V_{B}} \\
& =\sum_{j}-\mu_{j} v_{i j} \phi_{j} \frac{M_{F j}}{V_{B}} \\
& =\sum_{j}-\mu_{j} v_{i j} \phi_{j} X_{B j}
\end{aligned}
$$

where $A_{F}$ is the surface of the biofilm, $V_{B}$ is the volume of the bulk liquid compartment and $X_{B j}=M_{F j} / V_{B}$ is the concentration of particulate matter $j$ in the biofilm per unit of volume of the bulk liquid compartment. Hence, the problem of the different dimensions of the particulate components in both phases is taken care of directly and the explicit description of the mass transfer between

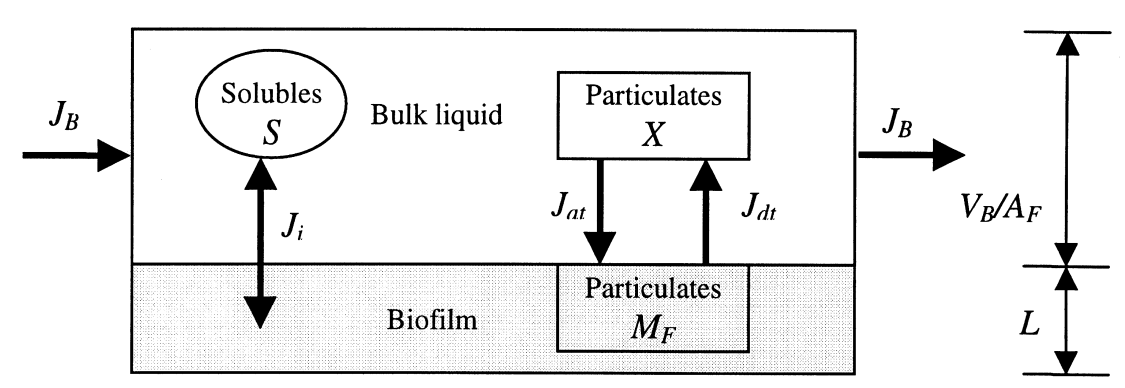

Fig. 6. Interaction processes between biofilm and bulk liquid components due to mass fluxes $J$. 
bulk liquid and biofilm must no longer be considered in the overall model.

\section{Physical interaction between biofilm and bulk liquid}

Attachment (flux $J_{\mathrm{at}}$ ) is addressing a number of physical processes where suspended matter is transported from the water phase to the biofilm compartment. The most important phenomena are processes such as sedimentation, inclusion or attachment which are generally described as a firstorder process with respect to the concentration of the particulate matter in the water phase. The reverse process of displacement is addressed as detachment (flux $J_{\mathrm{dt}}$ ). Detachment describes the material loss from the biofilm matrix and is frequently categorised into the phenomena erosion, sloughing and abrasion. In this paper no such distinction is made and all three phenomena are modelled as one. Following Horn and Hempel (1997b) the rate of biomass detachment $k_{d}$ is formulated here as being dependent on the velocity $u_{f}$ by which the biofilm surface moves relative to the substratum:

$$
\begin{array}{ll}
\text { if } \quad u_{f}<=0 & \text { then } \quad k_{\mathrm{d}}=0 \\
\text { if } \quad u_{f}>0 & \text { then } \quad k_{\mathrm{d}}=k_{\mathrm{dt}} u_{f}
\end{array}
$$

where $k_{\mathrm{d}}$ is the detachment rate, $u_{f}$ the velocity by which the surface of the biofilm moves perpendicular to the substratum and $k_{\mathrm{dt}}$ the detachment coefficient.

Description of biokinetic processes in the biofilmProcess matrix: an example

The biokinetic process description is straightforward once the fractions of the active biomass have been computed as previously outlined. Expressing the components in the biofilm as above in terms of concentrations with respect to the volume of the bulk liquid compartment $\left(X_{B j}\right)$ does not violate mass conservation principles. In the following illustrative example aerobic and anoxic growth of heterotrophs and aerobic growth of autotrophs is simulated, as well as hydrolysis and decay. It is important that the process description for biomass growth used here (Table 2) is consistent with the one used earlier for determining the active fractions (Table 1).

Here we follow as closely as possible the concepts formulated in the activated sludge model No. 1 (Henze et al., 1987) for the description of the biokinetic processes in the biofilm (Table 2). The main difference to other biofilm models (e.g. Wanner and Gujer, 1986; Wanner and Reichert, 1996; Horn and Hempel, 1997a,b) is the consistent application of the death-regeneration principle (Dold et al., 1980) together with the introduction of slowly biodegradable organic matter $\left(X_{S}\right)$ which is considered as a particulate component of the biofilm. The reasoning for choosing this approach is to allow both a consistent process description and wastewater charac-

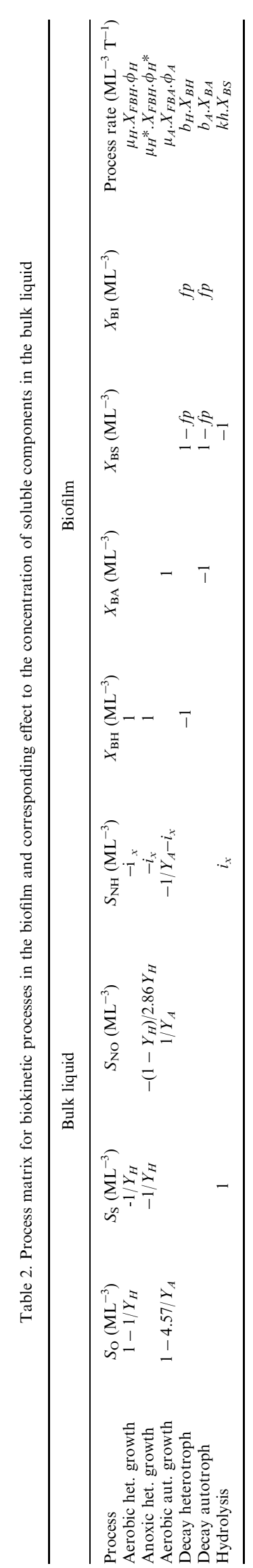


terisation for direct linkage of models of the various compartments in aquatic systems (e.g. Rauch et al., 1998). However, the choice of this concept is not mandatory for using the presented simplified biofilm model and it can be easily replaced.

However, with respect to the activated sludge model No. 1 (ASM1) some simplifications had to be implemented as a result of the active fraction concept. First of all bacterial growth is not expressed as a Monod-type reaction as done in ASM1 but instead as a first-order process with respect to the active fraction of the bacterial mass alone. This is due to the requirements for zeroorder in substrate kinetics and diffusion limitation. In ASM1 also the limitation of reactions with respect to oxygen and ammonia is expressed by Monod-type switching functions. Conveniently these functions can be dropped as all limitations are already considered in the active fraction concept.

In Table 2 it is postulated that hydrolysis is a first-order process with respect to the concentration of slowly biodegradable organic matter. This hypothesis is supported by experimental results from Janning et al. (1997). According to the assumptions underlying the model concept (assumption (5)) readily biodegradable organic matter from hydrolysis is modelled as being instantaneously transferred into the bulk liquid.

\section{APPLICATION OF THE MODEL}

Two examples will serve as illustration of the applicability of the simplified mixed-culture model. Both are long-term experiments in which growth and decay of microbial species in the biofilm have been investigated under different, well-defined environmental conditions. The first experiment (Horn and Hempel, 1997a) is concerned with the population dynamics in an autotrophic biofilm system over a period of 10 months. The experimental results have been used to calibrate the model with respect to the parameters of the autotrophic bacteria. The predictive power of the model is seen by the fact that no change of those calibrated parameter values was necessary to describe the second experiment, which is a 7-month investigation on growth and decay in an auto-/heterotrophic biofilm (Horn and Hempel, 1997b). Obviously, the lack of data in the first experiment caused that the par-

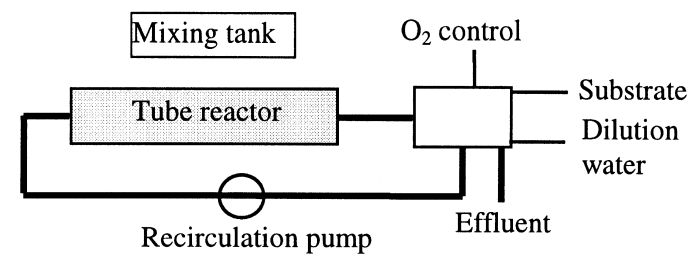

Fig. 7. Experimental set-up.
Table 3. Specification of the experimental set-up. (After: Horn and Hempel, 1997a,b)

\begin{tabular}{lll}
\hline Parameter & Experiment 1 & Experiment 2 \\
\hline Reactor length & $1.63 \mathrm{~m}$ & $1.42 \mathrm{~m}$ \\
Tube diameter & $0.016 \mathrm{~m}$ & $0.026 \mathrm{~m}$ \\
Biofilm surface area & $0.082 \mathrm{~m}^{2}$ & $0.116 \mathrm{~m}^{2}$ \\
Recirculation flow & $1.74 \mathrm{~m}^{3} \mathrm{~d}^{-1}$ & $3.67 \mathrm{~m}^{3} \mathrm{~d}^{-1}$ \\
Dilution water flow & $0.0035 \mathrm{~m}^{3} \mathrm{~d}^{-1}$ & $0.0035 \mathrm{~m}^{3} \mathrm{~d}^{-1}$ \\
Substrate solution flow & $0.0003 \mathrm{~m}^{3} \mathrm{~d}^{-1}$ & $0.0003 \mathrm{~m}^{3} \mathrm{~d}^{-1}$ \\
\hline
\end{tabular}

ameters describing heterotrophic bacteria conversion were calibrated for the second experiment only. For the sake of illustration both experiments are described briefly in the following. For a more detailed description the reader is referred to the original literature.

\section{Experimental set-up}

Both investigations used a nearly identical set-up which is characterised in Fig. 7 and Table 3. The very high recirculation rate makes it possible to model the biofilm tube reactor as a quasi completely mixed reactor. After inoculation with sludge from a wastewater treatment plant the biofilm was grown inside the darkened glass tube under a constant temperature of $20^{\circ} \mathrm{C}$. The measurements included (among others) biofilm thickness, mean biofilm density, flux of substrates and oxygen profiles. For determining detachment rates the suspended solids in the effluent have also been measured. Note the difference in measuring the substrate fluxes from the bulk liquid into the biofilm: the turnover rate of ammonia $\left(S_{\mathrm{NH}}\right)$ is determined in both experiments by measuring influent and effluent concentrations. For the evaluation of the maximum flux of glucose $S_{\mathrm{S}}$ in the second experiment (auto/heterotrophic biofilm) batch experiments had to be carried out. For this purpose, the reactor was rinsed with dilution water first and then the substrate concentration was set to $400 \mathrm{mg} / \mathrm{l}$ glucose. The decrease in glucose concentration in the reactor was monitored during $90 \mathrm{~min}$. The feeding with substrate and dilution water was turned down during the batch experiment, however the flow velocity in the reactor was kept constant in order to maintain complete mixing and mass transfer conditions.

\section{Autotrophic biofilm dynamics}

The long-term investigation of growth and decay of nitrifying bacteria has been divided into three phases (see Table 4 and Fig. 8) where in the first phase the exponential growth of the bacteria was explored, in the second the behaviour during starvation (no substrate loading) and in the third phase the restart of bacterial activity. Measurements of oxygen and substrate profiles (see Horn and Hempel, 1997a) revealed that after the initial growth period oxygen became the growth limiting substrate (approximately days 100 to 152 ). Note that the ammonia loading was reduced to $1.1 \mathrm{gm}^{-2} \mathrm{~d}^{-1}$ towards the end of phase 1 (days 152 to 163) which is not mentioned in the original literature (Horn, personal communication). As a consequence the bacterial growth was ammonia limited in this period. This also explains the peak (single point) in the ammonia flux at day 164 (Fig. 8). After restarting the ammonia loading at day 201 bacterial activity was immedi- 
Table 4. Substrate loading and oxygen concentration during the experiments. (After Horn and Hempel, 1997a,b)

\begin{tabular}{lccccc}
\hline Experiment & Phase & Day & $S_{\mathrm{S}}$ loading $\left(\mathrm{gm}^{-2} \mathrm{~d}^{-1}\right)$ & $S_{\mathrm{NH}}$ loading $\left(\mathrm{gm}^{-2} \mathrm{~d}^{-1}\right)$ & $\mathrm{O}_{2}\left(\mathrm{gm}^{-3}\right)$ \\
\hline 1 & 1 & $0-165$ & - & $3.0-4.0$ & 0.0 \\
& 2 & $166-200$ & - & 3.0 & 6.0 \\
2 & 3 & $201-296$ & 1.5 & 0.0 & $3.5-6.0$ \\
& 1 & $0-108$ & 0.0 & 3.0 & 6.0 \\
& 2 & $109-140$ & 1.0 & 3.0 & 6.0 \\
\hline
\end{tabular}

ately measured and increased linearly until the growth process was again oxygen limited. This oxygen limitation is also documented by the reduction in the measured flux when the oxygen concentration in the tube reactor was reduced from 6.0 to $3.5 \mathrm{mg} / 1$ from day 270 until the end of the experiment.

The parameters of the proposed model were assessed based on a combination of direct use of
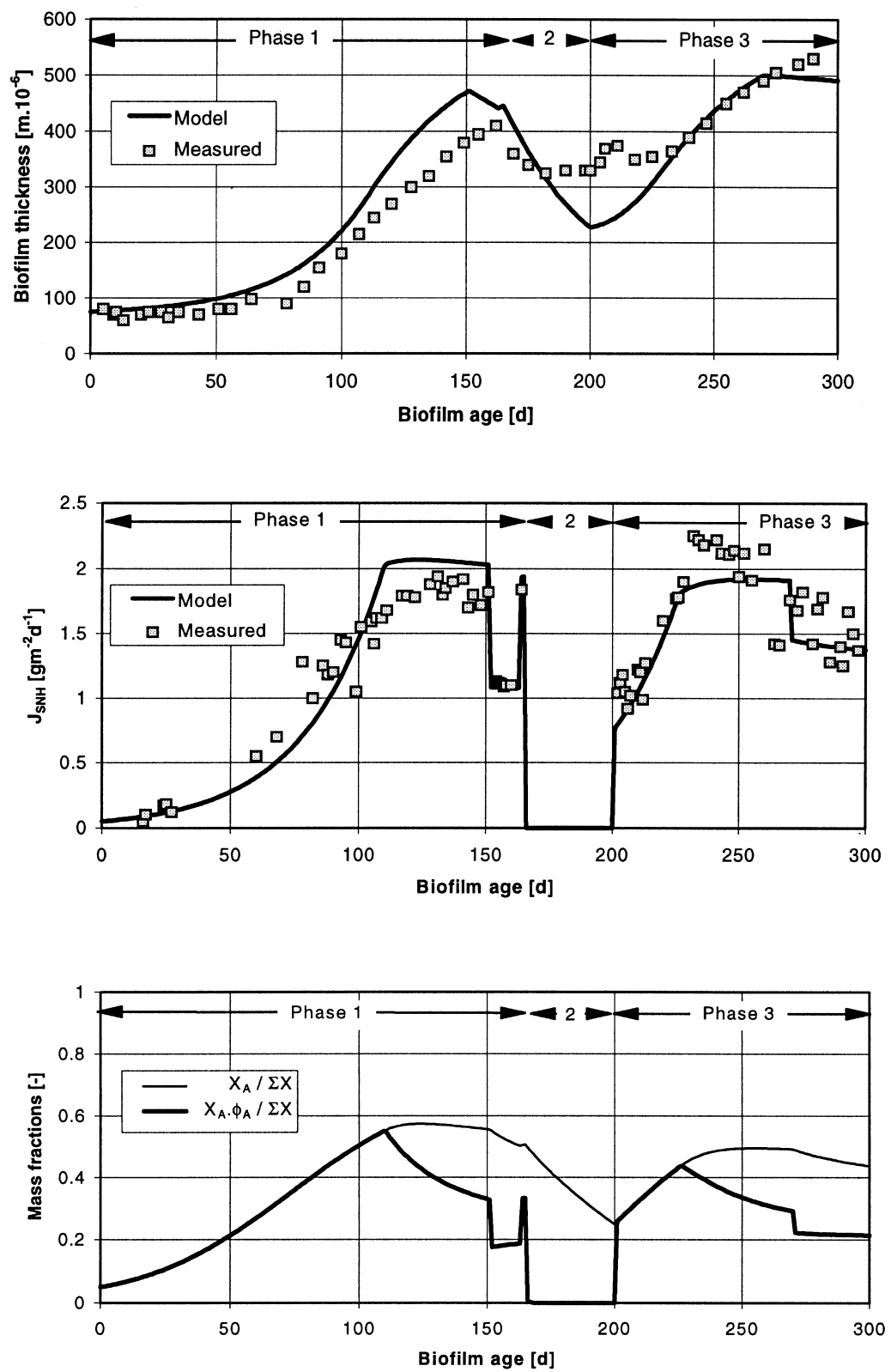

Fig. 8. Observed and simulated biofilm thickness (top) and ammonia flux (centre). Simulated mass fractions of autotrophic bacteria together with active amount (bottom). 
Table 5. Parameters used in the modelling predictions

\begin{tabular}{llll}
\hline Parameter & Exp. 1 & Exp. 2 & Estimated \\
\hline$\mu_{H}(1 / \mathrm{d})$ & & 0.8 & \\
$Y_{H}(\mathrm{gCOD} / \mathrm{gCOD})$ & & 0.085 & \\
$b_{H}(1 / \mathrm{d})$ & & 0.015 & \\
$\mu_{A}(1 / \mathrm{d})$ & 0.075 & 0.075 & Yes \\
$Y_{A}(\mathrm{gCOD} / \mathrm{gN})$ & 0.115 & 0.115 & \\
$b_{A}(1 / \mathrm{d})$ & 0.04 & 0.04 & \\
$k h(1 / \mathrm{d})$ & 0.08 & 0.08 & \\
$i_{x}(\mathrm{gN} / \mathrm{gCOD})$ & 0.08 & 0.08 & \\
$f \mathrm{p}(-)$ & 0.08 & 0.08 & \\
$k_{\mathrm{dt}}(-)$ & 0.1 & 0.1 & \\
$D_{S o}\left(\mathrm{~cm}^{2} / \mathrm{d}\right)$ & 2.1 & 2.1 & \\
$D_{S s}\left(\mathrm{~cm}^{2} / \mathrm{d}\right)$ & 0.58 & 0.58 & \\
$D_{S n h}\left(\mathrm{~cm}^{2} / \mathrm{d}\right)$ & 1.8 & 1.8 & \\
$\rho_{m}\left(\mathrm{~kg} / \mathrm{m}^{3}\right)$ & 20 & 25 & \\
\hline
\end{tabular}

measured data, literature data and calibration using non-linear optimisation techniques. Mean biofilm density $\rho_{m}$ was measured several times during the experimental period and the mean value of those measurements was implemented in the model. Horn and Hempel (1997b) identified the detachment rate directly by comparing measured and simulated values of suspended solids in the effluent. Here we consistently used their findings of the second experiment for the detachment coefficient $k_{\mathrm{dt}}$. Diffusion coefficients and stoichiometric coefficients were also taken directly from literature (Horn and Hempel, 1997b). All other parameter $\left(\mu_{A}, Y_{A}, b_{A}, k_{h}\right)$ values and the initial conditions for volume fractions and biofilm thickness were determined by minimisation of the error when comparing simulation with experimental results. The fit was carried out by using a genetic algorithm (Goldberg, 1989) for non-linear optimisation. The calibrated set of model parameters for both experiments is listed in Table 5 and initial conditions in Table 6.

In Fig. 8 the simulated ammonia flux and biofilm thickness is compared against the measurements. It is apparent that the ammonia turnover rate predictions of the model are excellent and in fact comparable to the results obtained by using the much more refined model of Horn and Hempel (1997a). In general also the biofilm thickness is simulated very well by the simplified mixed-culture model but a deviation with respect to biomass loss during starvation (phase 2) is observed. Figure 8 also depicts the profile of the simulated volume fraction of the nitrifying bacteria within the solid phase $\left(\varepsilon_{S A}\right)$ together with the active fraction of the component. Substrate

Table 6. Initial conditions used in the modelling predictions

\begin{tabular}{lcc}
\hline Parameter & Exp. 1 & Exp. 2 \\
\hline$L_{\text {ini }}\left(\mathrm{m} \times 10^{-6}\right)$ & 75 & 40 \\
$M_{A} / \Sigma M_{\text {ini }}(-)$ & 0.05 & 0.05 \\
$M_{H} / \Sigma M_{\text {ini }}(-)$ & & 0.00005 \\
\hline
\end{tabular}

limitation occurs as soon as the total amount of species present is no longer active, i.e. participating in the conversion process. The predicted limitations-for oxygen starting at day 110 and day 227 and for ammonia at days 151 to 163 -correspond very well with the measured substrate and oxygen profiles in Horn and Hempel (1997a). However, one should note that the simulation results with respect to the active fractions assume a uniform distribution of the particulate components, whereas probably a spatial distribution exists. Notwithstanding this discrepancy, the model was able to predict the general behaviour of biomass growth and decay over a long period.

\section{Autotrophic-heterotrophic biofilm dynamics}

The investigation of the dynamics of growth and decay of a mixed-culture biofilm was again divided into three phases (Table 4 and Fig. 9) comparable to the first experiment. The first phase was concerned with the growth of the heterotrophic bacteria group only. Substrate (glucose) loading was the limiting factor for growth during this period which made the earlier described batch experiments necessary to predict the maximum turnover rates for glucose. Again the second phase was a period of starvation for the biomass where no substrate was fed. In phase three glucose and ammonia were fed simultaneously to the biofilm thus exploring the growth of two species and their competition for oxygen and space.

Figure 9 compares the measurements with predicted values of biofilm thickness, ammonia flux and maximum glucose flux obtained by the simplified mixed-culture biofilm model. In order to test the predictive power of the model all parameter values already calibrated in the first experiment were used here without any adjustment. Hence, calibration was performed only for the parameters related to the dynamics of the heterotrophic bacteria $\left(\mu_{H}, Y_{H}, b_{H}\right)$ and for the initial conditions. The mean biofilm density has been obtained again from direct measurements.

As in the first experiment, the simplified mixedculture biofilm model was able to replicate the overall dynamics very closely. For the maximum glucose flux a maximum deviation of $22 \%$ between the simulated and recorded values was found at the end of phase two. This difference is probably due to both the heterogeneity of the biofilm and the complex behaviour with respect to lysis, death and maintenance. Although this is discussed at length by Horn and Hempel (1997b) their refined model also failed in accurate glucose flux predictions. The linear increase in ammonia flux in the third phase is described very well with the proposed model showing only a small discrepancy of $14 \%$ at the end of the experiment. Note that the start of the growth of the autotrophic bacteria species was implemented as an initial condition of the model specified for day 

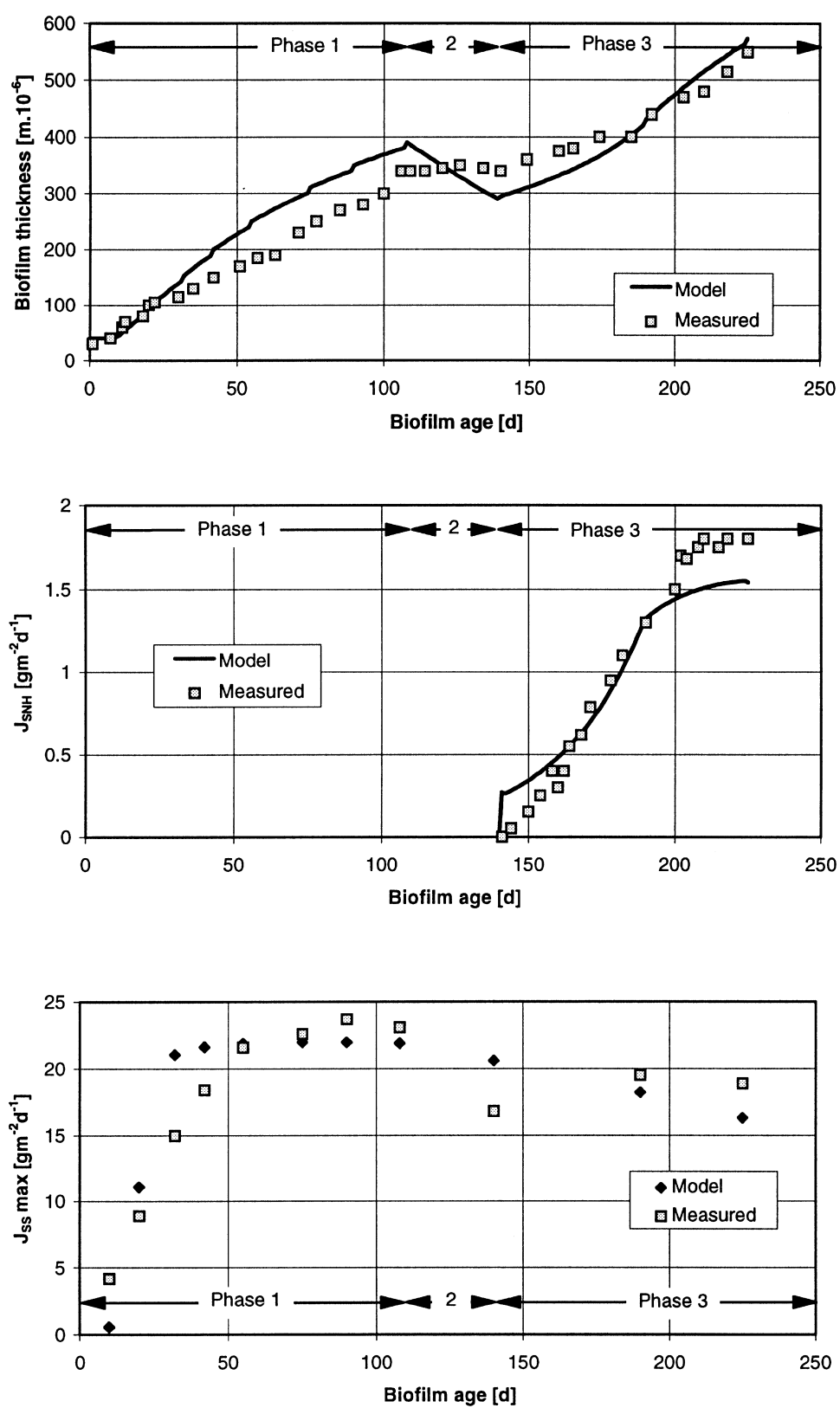

Fig. 9. Observed and simulated biofilm thickness (top), ammonia flux (centre) and maximum glucose flux measured in the batch experiment (bottom).

141 ( $X_{B A}$ being zero prior to that time instant), which corresponds to the approach of Horn and Hempel (1997b).

\section{Discussion}

The limitation of conversion processes due to the diffusion of soluble components $\left(S_{\mathrm{O}}, S_{\mathrm{NH}}, S_{\mathrm{S}}\right)$ is one of the major aspects in the investigated experiments. It is apparent from Figs 8 and 9 that the proposed active fraction concept is able to describe substrate limitation according to reality. In spite of the fact that the spatial distribution of the species within the biofilm is neglected, the principles of the active fraction concept proved to be applicable for the simulation of biofilm dynamics.

In both simulations the model predicted a loss of biomass activity that was not observed in the same magnitude during the experiment. The reason for this discrepancy is not the chosen description of biomass detachment but the implemented process description for modelling endogenous respiration and death. The deat -regeneration concept assumes 
that only a fraction $\left(f_{p}\right)$ of the biomass undergoes inactivation whereas the rest is first converted to slowly biodegradable organic matter $\left(X_{S}\right)$, subsequently hydrolysed and then converted back to biomass. During the starvation period the bacterial growth process is stopped. Hence, thickness decreases due to the hydrolysis of the slowly biodegradable organic matter.

Some of the parameters used in the model have been either taken directly from measurements $\left(\phi_{m}\right)$ or without change from Horn and Hempel (1997b) $\left(D_{i}, k_{\mathrm{dt}}\right)$. Parameters concerning the approaches from ASM1 were taken from Henze et al. (1987) $\left(i_{x}, f p\right)$. All other parameters had to be adjusted in order to fit the experimental results. Although most of the values found (Table 5) are well within the range of recorded data (Kissel et al., 1984; Wanner and Gujer, 1986; Arvin and Harremoës, 1990; Horn and Hempel, 1997 a, b) a difference is seen for the maximum growth rate and the yield coefficient of heterotrophic bacteria. Although both values deviate individually from commonly used parameter values, the relation $\mu_{H} / Y_{H}$ (expressing the maximum specific substrate conversion rate) is again a standard value. The difference in the parameter values is probably due to the use of the death regeneration concept as this leads sometimes to special parameter combinations for the description of decay. This problem is well known in activated sludge modeling but might be even more pronounced here.

\section{CONCLUSIONS}

We presented a simplified mixed-culture biofilm model and discussed the application of the model for two long-term experiments described in the literature. The basic idea of the proposed model is to decrease model complexity in order to allow fast but sufficiently accurate simulation of biofilm dynamics. This is achieved by decoupling the calculation of substrate diffusion into the biofilm from the computation of the conversion processes in the biofilm. The first step of the procedure assumes a pseudo-steady-state situation with respect to the diffusion and solves the problem analytically. The result of this first step is an estimate on the fraction of each biomass species that is active in the present situation. In the second step of the procedure all conversion processes within the biofilm are computed dynamically. The bacterial species are assumed to be homogeneously distributed as in an ideally mixed tank reactor but with only a certain fraction of each species active.

The critical assumption made in the model is the homogeneous distribution of the bacterial species. However, as seen from Fig. 9 this simplifying assumption does not seem to affect the descriptive ability of the model for a mixed-culture biofilm sys- tem. Meanwhile, the avoidance of partial differential equations for computation of the biofilm dynamics by using the two-step procedure instead results in a significant reduction of the model structure complexity as compared to state-of-the-art mixed-culture biofilm models. This feature allows a direct implementation of standard biokinetic conversion models as used in activated sludge modelling. We conclude that the simplified mixed-culture biofilm model is an attractive tool when emphasis is put more on fast predictions of system behaviour than on detailed understanding.

Acknowledgements-Financial support for this work was provided by the Austrian Government and the Fund for Scientific Research, Flanders (Belgium) (F.W.O.). The second author is Research Assistant of the Fund for Scientific Research, Flanders (Belgium).

\section{REFERENCES}

Arvin E. and Harremoës P. 1990. Concepts and models for biofilm reactor performance. Wat. Sci. Techn. 22 (12) $171-192$.

Bishop P. (1997) Biofilm structure and kinetics. Wat. Sci. Techn. 36 (1), 287-294.

Christiansen P., Hollesen L. and Harremoës P. (1995) Liquid film diffusion on reaction rate in submerged biofilters. Wat. Res. 29 (3), 947-952.

Dold P.L., Ekama G.A. and Marais GvR. (1980) A general model for the activated sludge process. Prog. Water Techn. 12, 47-77.

Goldberg D. E. (1989) Genetic algorithms in Search, Optimization and Machine Learning. Addison-Wesley, Reading, Mass.

Harremoës P. (1978) Biofilm kinetics. In: Water Pollution Microbiology. Mitchell (ed.) Vol. 2. pp. 71-109. Wiley, N.Y.

Henze M., Grady C. P. L., Gujer W., Marais GvR and Matsuo T. (1987) Activated sludge model No. 1. IAWQ, London, ISSN: 1010-707X.

Horn H. and Hempel D. C. (1997a) Substrate utilization and mass transfer in an autotrophic biofilm system: Experimental results and numerical simulation. Biotech. and Bioeng. 53 (4), 363-371.

Horn H. and Hempel D. C. (1997b) Growth and decay in an auto-/heterotrophic biofilm. Wat. Res. 31 (9), 22432252 .

Janning K. F., Mesterton K. and Harremoës P. (1997) Hydrolysis and degradation of filtrated organic particulates in a biofilm reactor under anoxic and aerobic conditions. Wat. Sci. Techn. 36 (1), 279-286.

Kissel J. C., McCarty P. L. and Street R. L. (1984) Numerical simulation of mixed-culture biofilm. J. Env. Engin. 110 (2), 393-412.

Lewandowski Z., Stoodley P., Altobelli S. and Fukushima E. (1994) Hydrodynamics and kinetics in biofilm systems - recent advances and new problems. Wat. Sci. Techn. 29 (1011), 223-229.

Mueller J. A., Paquin P. and Famularo J. (1980) Nitrification in rotating biological rotators. Water Pollut. Control Fed. 52 (4), 688-710.

Rauch W. and Vanrolleghem P. A. (1997) A dynamic biofilm model for simulation of wastewater treatment processes and benthic activity in rivers. Technical report. BIOMATH Department, University Gent, Belgium.

Rauch W., Aalderink H., Krebs K., Schilling W. and Vanrolleghem P. (1998) Requirements for integrated 
wastewater models - driven by receiving water objectives. Wat. Sci. Techn.

Wanner O. and Gujer W. (1986) A multispecies biofilm model. Biotech. and Bioeng. 28, 314-328.

Wanner O. and Reichert P. (1996) Mathematical modeling of mixed-culture biofilms. Biotech. and Bioeng. 49 (2), 172-184.
Williamson K. and McCarty P. L. (1976) A model of substrate utilization by bacterial films. Water Pollut. Control Fed. 48 (1), 9-24.

Zhang T. C. and Bishop P. L. (1994) Density, porosity and pore structure of biofilms. Wat. Res. 28 (11), 2267 2277. 